\title{
Almost Surely Exponential Stability of Numerical Solutions for Stochastic Pantograph Equations
}

\author{
Shaobo Zhou \\ School of Mathematics and Statistics, Huazhong University of Science and Technology, Wuhan 430074, China \\ Correspondence should be addressed to Shaobo Zhou; zhoushaobowls@hust.edu.cn
}

Received 25 September 2013; Revised 10 April 2014; Accepted 10 April 2014; Published 3 June 2014

Academic Editor: Abdon Atangana

Copyright ( 2014 Shaobo Zhou. This is an open access article distributed under the Creative Commons Attribution License, which permits unrestricted use, distribution, and reproduction in any medium, provided the original work is properly cited.

\begin{abstract}
Our effort is to develop a criterion on almost surely exponential stability of numerical solution to stochastic pantograph differential equations, with the help of the discrete semimartingale convergence theorem and the technique used in stable analysis of the exact solution. We will prove that the Euler-Maruyama (EM) method can preserve almost surely exponential stability of stochastic pantograph differential equations under the linear growth conditions. And the backward EM method can reproduce almost surely exponential stability for highly nonlinear stochastic pantograph differential equations. A highly nonlinear example is provided to illustrate the main theory.
\end{abstract}

\section{Introduction}

Stochastic unbounded delay systems play an important role in a variety of application areas, including biology, epidemiology, mechanic, economics, and finance. The systems provide powerful models, such as infinite delay Kolmogorov-type systems in mathematic biology [1-4], stochastic neural networks [5-8], and stochastic pantograph equations in science and engineering. The pantograph equation which is a very special unbounded delay equation was used by Ockendon and Tayler [9] in 1971 to study how the electric current is collected by the pantograph of an electric locomotive, from where it gets its name. Such systems have received an increasing attention (see e.g., $[3,10-13]$ ).

Unfortunately, most of stochastic differential equations cannot be solved explicitly. Especially, explicit solutions can rarely be obtained for nonlinear stochastic pantograph equations, so numerical methods have recently received more and more attention (see $[6,8,13-15])$. Most of research efforts have been devoted to the convergence and mean-square stability of various numerical methods for the linear delay systems [14, 16-19]. Recently, several authors were devoted to the convergence in probability of the Euler-Maruyama (EM) method for the nonlinear delay systems. For example,
Mao [20] and Milošević [21] developed the convergence in probability of the EM approximate solution for nonlinear SDDE and neutral SDDE under the Khasminskii-type conditions, respectively. Zhou et al. [22, 23] established the convergence in probability of the EM approximation for neutral stochastic functional differential equation under the polynomial growth conditions.

Stability theory of numerical solution is one key problem in numerical analysis. Compared with the convergence, the study on stability of numerical methods for delay systems is relatively scarce due to infinite time-delay that is often the source of instability. Research efforts have been devoted to various stabilities of numerical methods for SDEs. For example, Higham et al. [15, 24-26] investigated stability of numerical methods for SDEs. Pang et al. [27] showed that the EM discretization can capture almost surely and moment exponential stability for all sufficiently small time-step under appropriate conditions for linear hybrid SDE. Mao et al. [28] showed that the backward EM method can reproduce almost surely exponential stability of nonlinear hybrid SDE. Mao and Szpruch [29] developed almost surely asymptotic properties of implicit numerical methods for nonlinear SDEs, via a stochastic version of LaSalle principle. However, the stability of numerical method for highly nonlinear stochastic delay 
system is less studied, due to time-delay that is often the source of instability (see $[2,30,31]$ ), which is the main topic of the present paper.

The stability of numerical method is inspired by Wu et al.'s paper [32], in which they first studied that the backward EM method can reproduce almost surely exponential stability of nonlinear stochastic differential delay system

$$
\mathrm{d} x(t)=f(x(t), x(t-\tau)) \mathrm{d} t+g(x(t), x(t-\tau)) \mathrm{d} w(t),
$$

by using of the discrete semi-martingale convergence theorem, under the following conditions

$$
\begin{gathered}
2 x^{T} f(x, 0) \leq-\lambda_{1}|x|^{2} \\
|f(x, y)-f(x, 0)|^{2} \leq \lambda_{2}|y|^{2}, \\
|g(x, y)|^{2} \leq \lambda_{3}|x|^{2}+\lambda_{4}|y|^{2} .
\end{gathered}
$$

Wu et al.'s work is a very important contribution to numerical SDDE theory. Certainly, we also see that the theory imposes the one-sided linear growth on the drift coefficient (i.e., (3)) and the linear growth on the diffusion coefficient (i.e., (4)), which are rather strong so that many highly nonlinear SDDEs are excluded. Recently, Zhou et al. [33] studied exponential stability of stochastic functional differential equation under polynomial growth condition. To the best of author's knowledge, there is no work on stability of numerical method for stochastic pantograph differential equations. In the paper, our effort is to develop a new criterion on almost surely exponential stability of numerical solution to stochastic pantograph differential equations, with the help of the discrete semimartingale convergence theorem and the technique used in stability analysis of the exact solution. We will prove that the Euler-Maruyama method can preserve the almost surely exponential stability of stochastic pantograph differential equations under the linear growth conditions. The backward EM method can preserve almost surely exponential stability for highly nonlinear stochastic pantograph differential equations under sufficiently small step size.

\section{The Global Solution}

Throughout this paper, unless otherwise specified, let $|x|$ be the Euclidean norm in $x \in \mathbb{R}^{n}$. If $A$ is a vector or matrix, its transpose is denoted by $A^{T}$. If $A$ is a matrix, its trace norm is denoted by $|A|=\sqrt{\operatorname{trace}\left(A^{T} A\right)}$, while its operator norm is denoted by $\|A\|=\sup \{|A x|:|x|=1\}$. Let $\left(\Omega, \mathscr{F},\left\{\mathscr{F}_{t}\right\}_{t \geq 0}, P\right)$ be a complete probability space with a filtration $\left\{\mathscr{F}_{t}\right\}_{t \geq 0}$, satisfying the usual conditions (i.e., it is increasing and right continuous and $\mathscr{F}_{0}$ contains all $P$-null sets). Let $w(t)=\left(w_{1}(t), w_{2}(t), \ldots, w_{m}(t)\right)$ be $m$-dimensional Brownian motion. tion

Consider an $n$-dimensional stochastic pantograph equa-

$$
\mathrm{d} x(t)=f(x(t), x(q t)) \mathrm{d} t+g(x(t), x(q t)) \mathrm{d} w(t),
$$

on $t \geq 0$ with the initial data $\left.x_{0} \in R^{n}\right) ; f: \mathbb{R}^{n} \times \mathbb{R}^{n} \rightarrow \mathbb{R}^{n}, g$ : $\mathbb{R}^{n} \times \mathbb{R}^{n} \rightarrow \mathbb{R}^{n}$ are locally Lipschitz continuous.

(H1) The Local Lipschitz Condition. For each integer $R \geq 1$, there exists a positive constant $K_{R}$ such that

$$
\begin{aligned}
\left|f\left(x_{1}, y_{1}\right)-f\left(x_{2}, y_{2}\right)\right| & \vee\left|g\left(x_{1}, y_{1}\right)-g\left(x_{2}, y_{2}\right)\right| \\
\leq & K_{R}\left(\left|x_{1}-x_{2}\right|+\left|y_{1}-y_{2}\right|\right)
\end{aligned}
$$

for all $x_{k}, y_{k} \in \mathbb{R}^{n}$ with $\left|x_{k}\right| \vee\left|y_{k}\right| \leq R(k=1,2)$.

(H2) The Polynomial Growth Conditions. For all $x \in \mathbb{R}^{n}$, there exist positive constants $\alpha, \beta, \gamma, a, \bar{a}, \tilde{a}, b, \bar{b}, \tilde{b}$ such that

$$
\begin{aligned}
& \langle x(s), f(x(s), x(q s))\rangle \\
& \quad \leq-a|x(s)|^{\alpha+2}+\bar{a}\left(|x(q s)|^{\beta+2}+|x(s)|^{\beta+2}\right)-\tilde{a}|x(s)|^{2},
\end{aligned}
$$

$$
|g(x(s), x(q s))|^{2} \leq b|x(s)|^{\gamma+2}+\bar{b}|x(q s)|^{\gamma+2}+\widetilde{b}|x(s)|^{2} .
$$

Lemma 1 (see $[1])$. Let $\alpha, b>0, \kappa(x) \in C\left(\mathbb{R}_{+} ; \mathbb{R}\right)$; if $\kappa(x)=$ $o\left(|x|^{\alpha}\right)(|x| \rightarrow \infty)$, then

$$
\sup _{x \in \mathbb{R}_{+}}\left[\kappa(x)-b|x|^{\alpha}\right]<\infty .
$$

Theorem 2. Let (H1) and (H2) hold with $\alpha \geq \beta \vee \gamma, 2 \widetilde{a}>\widetilde{b}$, $2 a>2 \bar{a}(1+1 / q)+b+\bar{b} / q$. Then for any initial data $x_{0}$, there almost surely exists a unique global solution $x(t)$ to (5) on $t \geq 0$.

Proof. Under (H1), applying the standing truncation technique to (5) for any initial data $x_{0}$, there exists a unique maximal local strong solution $0<t<v_{e}$, where $v_{e}$ is the explosion time. To show this solution is global, we only need to show that $\nu_{e}=\infty$ a.s. Let $k_{0}>0$ be sufficiently large such that $k_{0}>\left|x_{0}\right|$. For each integer $k \geq k_{0}$, define the stopping time

$$
v_{k}=\inf \left\{t \in\left[0, v_{e}\right):|x(t)| \geq k\right\}, \quad k \in N,
$$

where throughout this paper, we set inf $\emptyset=\infty$ (as usual, $\emptyset$ $=$ the empty set). By the definition of the stopping time $v_{k}$, it is obvious that $v_{k}$ is an increasing function with $k$, so $v_{k} \rightarrow$ $v_{\infty} \leq v_{e}(k \rightarrow \infty)$ a.s. If we can show that $v_{\infty}=\infty$ a.s., then $v_{e}=\infty$ a.s. which implies that $x(t)$ is global. In other words, we only prove that $P\left(v_{k} \leq t\right) \rightarrow 0(k \rightarrow \infty, t>0)$. Define $V(x)=|x|^{2}$, by $P\left(v_{k} \leq t\right) V\left(x\left(v_{k}\right)\right) \leq E V\left(x\left(t \wedge v_{k}\right)\right)$; we only need to prove that $E V\left(x\left(t \wedge v_{k}\right)\right)<+\infty$ according to $V\left(x\left(v_{k}\right)\right)=\left|x\left(v_{k}\right)\right|^{2}=k^{2} \rightarrow \infty$. For $\varepsilon>0$, by the Itô formula, we have

$$
\begin{aligned}
V\left(x\left(t \wedge v_{k}\right)\right)=V(x(0))+\int_{0}^{t \wedge v_{k}}( & 2 x^{T} f(x(s), x(q s)) \\
& \left.+|g(x(s), x(q s))|^{2}\right) \mathrm{d} s
\end{aligned}
$$$$
+M(t)
$$ 
where $M(t)=\int_{0}^{t \wedge v_{k}} \mathrm{e}^{\varepsilon s} V_{x}(x(s)) g(x(s), x(q s)) \mathrm{d} w(s) \mathrm{d} s$ is a real-valued continuous local martingale with $M(0)=0$. By (7) and (8), we may also estimate

$$
\begin{aligned}
2 x^{T} f & (x(s), x(q s))+|g(x(s), x(q s))|^{2} \\
\leq & -2 a|x(s)|^{\alpha+2}+2 \bar{a}\left(|x(q s)|^{\beta+2}+|x(s)|^{\beta+2}\right)-2 \widetilde{a}|x(s)|^{2} \\
& +b|x(s)|^{\gamma+2}+\bar{b}|x(q s)|^{\gamma+2}+\widetilde{b}|x(s)|^{2} \\
\leq & \frac{2 \bar{a}}{q}\left(q|x(q s)|^{\beta+2}-|x(s)|^{\beta+2}\right) \\
& +\frac{\bar{b}}{q}\left(q|x(q s)|^{\gamma+2}-|x(s)|^{\gamma+2}\right)-2 a|x(s)|^{\alpha+2} \\
& -(2 \widetilde{a}-\widetilde{b})|x(s)|^{2}+2 \bar{a}\left(1+\frac{1}{q}\right)|x(s)|^{\beta+2} \\
& +\left(b+\frac{\bar{b}}{q}\right)|x(s)|^{\gamma+2} .
\end{aligned}
$$

Denoted by

$$
\begin{aligned}
I(x(s))= & (2 \widetilde{a}-\tilde{b})|x(s)|^{2}+2 a|x(s)|^{\alpha+2} \\
& -2 \bar{a}\left(1+\frac{1}{q}\right)|x(s)|^{\beta+2}-\left(b+\frac{\bar{b}}{q}\right)|x(s)|^{\gamma+2} .
\end{aligned}
$$

Noting that $2 \widetilde{a}>\widetilde{b}, 2 a>2 \bar{a}(1+1 / q)+b+\bar{b} / q, \alpha \geq \beta \vee$ $\gamma$, by Lemma 1 , there exists a positive $c_{0}$ such that $I(x(s)) \geq$ $c_{0}|x(s)|^{2}$. Substitution for this and (12) into (11) yields

$$
\begin{aligned}
V\left(x\left(t \wedge v_{k}\right)\right) \leq & V(x(0))+M(t) \\
& +\frac{2 \bar{a}}{q} \int_{0}^{t \wedge v_{k}}\left(q|x(q s)|^{\beta+2}-|x(s)|^{\beta+2}\right) \mathrm{d} s \\
& +\frac{\bar{b}}{q} \int_{0}^{t \wedge v_{k}}\left(q|x(q s)|^{\gamma+2}-|x(s)|^{\gamma+2}\right) \mathrm{d} s \\
& -c_{0} \int_{0}^{t \wedge v_{k}}|x(s)|^{2} \mathrm{~d} s .
\end{aligned}
$$

Making use of the property of the integral, we may estimate

$$
\begin{aligned}
\int_{0}^{t} \mathrm{e}^{\varepsilon s} & \left(q|x(q s)|^{\beta+2}-|x(s)|^{\beta+2}\right) \mathrm{d} s \\
& =\int_{0}^{t}\left(q|x(q s)|^{\beta+2}-|x(s)|^{\beta+2}\right) \mathrm{d} s \\
& \leq \int_{0}^{q t}|x(s)|^{\beta+2} \mathrm{~d} s-\int_{0}^{t}|x(s)|^{\beta+2} \mathrm{~d} s \\
& =-\int_{q t}^{t}|x(s)|^{\beta+2} \mathrm{~d} s .
\end{aligned}
$$

Similarly,

$$
\int_{0}^{t}\left(q|x(q s)|^{\gamma+2}-|x(s)|^{\gamma+2}\right) \mathrm{d} s \leq-\int_{q t}^{t}|x(s)|^{\gamma+2} \mathrm{~d} s .
$$

Substituting (15) and (16) into (14) and taking expectation yield

$$
\begin{aligned}
\mathbb{E} V\left(x\left(t \wedge v_{k}\right)\right) \leq & \mathbb{E}\|\xi\|^{2}-\frac{2 \bar{a}}{q} \int_{q t}^{t \wedge v_{k}}|x(s)|^{\beta+2} \mathrm{~d} s \\
& -\frac{\bar{b}}{q} \int_{q\left(t \wedge v_{k}\right)}^{t \wedge v_{k}}|x(s)|^{\gamma+2} \mathrm{ds} \\
& -c_{0} \int_{0}^{t \wedge v_{k}}|x(s)|^{2} \mathrm{~d} s \leq \mathbb{E}\|\xi\|^{2},
\end{aligned}
$$

which implies that there exists almost surely a unique global solution. The proof is complete.

For stability, we need to impose a stronger condition on the coefficients as follows.

(H3) The Polynomial Growth Conditions. For all $x \in \mathbb{R}^{n}$, there exist positive constants $\alpha, \beta, \gamma, a, \bar{a}, \widetilde{a}, b, \bar{b}, \widetilde{b}$, and $\varepsilon$ with $\varepsilon \leq$ $2 \widetilde{a}-\widetilde{b}$ such that

$$
\begin{aligned}
\langle x & (s), f(x(s), x(q s))\rangle \\
\leq & -a|x(s)|^{\alpha+2}+\bar{a}\left(\mathrm{e}^{-(1-q) \varepsilon s}|x(q s)|^{\beta+2}+|x(s)|^{\beta+2}\right) \\
& -\widetilde{a}|x(s)|^{2}, \\
|g(x(s), x(q s))|^{2} & \leq b|x(s)|^{\gamma+2}+\bar{b} \mathrm{e}^{-(1-q) s s}|x(q s)|^{\gamma+2}+\widetilde{b}|x(s)|^{2} .
\end{aligned}
$$

Theorem 3. Let (H1) and (H3) hold with $\alpha \geq \beta \vee \gamma, 2 \widetilde{a}>\widetilde{b}$, $2 a>2 \bar{a}(1+1 / q)+b+\bar{b} / q$. Then for any initial data $x_{0}$, the solution $x(t)$ is pth moment and almost surely exponentially stable; that is,

$$
\lim \sup _{t \rightarrow \infty} \frac{1}{t} \log |x(t)| \leq-\frac{\varepsilon}{2},
$$

where $\varepsilon \leq 2 \widetilde{a}-\widetilde{b}$.

Proof. Clearly, (H3) implies that (H2) by letting $\varepsilon=0$, so there exists a unique global solution. Define $V(x)=|x|^{2}$, for any $\varepsilon>0$; by the Itô formula, we have

$$
\begin{aligned}
\mathrm{e}^{\varepsilon t} V(x(t))= & V(x(0))+\int_{0}^{t} \mathrm{e}^{\varepsilon s}(L V(x(s), x(q s)) \\
& +\varepsilon V(x(s))) \mathrm{d} s+\bar{M}(t),
\end{aligned}
$$


where $\bar{M}(t)=\int_{0}^{t} \mathrm{e}^{\varepsilon s} V_{x}(x(s)) g(x(s), x(q s)) \mathrm{d} w(s) \mathrm{d} s$ is a realvalued continuous local martingale with $M(0)=0$. By (18) and (19), we may also estimate

$$
\begin{aligned}
L V(x(s) & , x(q s))+\varepsilon V(x(s)) \\
\leq & -2 a|x(s)|^{\alpha+2}+2 \bar{a}\left(\mathrm{e}^{-(1-q) \varepsilon s}|x(q s)|^{\beta+2}+|x(s)|^{\beta+2}\right) \\
& -2 \widetilde{a}|x(s)|^{2}+b|x(s)|^{\gamma+2}+\bar{b} \mathrm{e}^{-(1-q) \varepsilon s}|x(q s)|^{\gamma+2} \\
& +\widetilde{b}|x(s)|^{2}+\varepsilon|x(s)|^{2} \\
\leq & \frac{2 \bar{a}}{q}\left(q \mathrm{e}^{-(1-q) \varepsilon s}|x(q s)|^{\beta+2}-|x(s)|^{\beta+2}\right) \\
& +\frac{\bar{b}}{q}\left(q \mathrm{e}^{-(1-q) \varepsilon s}|x(q s)|^{\gamma+2}-|x(s)|^{\gamma+2}\right)-2 a|x(s)|^{\alpha+2} \\
& -(2 \widetilde{a}-\widetilde{b}-\varepsilon)|x(s)|^{2}+2 \bar{a}\left(1+\frac{1}{q}\right)|x(s)|^{\beta+2} \\
& +\left(b+\frac{\bar{b}}{q}\right)|x(s)|^{\gamma+2} .
\end{aligned}
$$

Denoted by

$$
\begin{aligned}
I(x(s))= & (2 \widetilde{a}-\widetilde{b}-\varepsilon)|x(s)|^{2}+2 a|x(s)|^{\alpha+2} \\
& -2 \bar{a}\left(1+\frac{1}{q}\right)|x(s)|^{\beta+2}-\left(b+\frac{\bar{b}}{q}\right)|x(s)|^{\gamma+2} .
\end{aligned}
$$

Noting that $2 \widetilde{a}>\widetilde{b}, 2 a>2 \bar{a}(1+1 / q)+b+\bar{b} / q, \varepsilon<2 \widetilde{a}-\widetilde{b}$, $\alpha \geq \beta \vee \gamma$, by Lemma 1 , there exists a positive $\bar{c}_{0}$ such that $I(x(s)) \geq \bar{c}_{0}|x(s)|^{2}$. Substitution for this and (22) into (21) yields

$$
\begin{aligned}
\mathrm{e}^{\varepsilon t} V(x(t)) \leq & V(x(0))+\bar{M}(t) \\
& +\frac{2 \bar{a}}{q} \int_{0}^{t} \mathrm{e}^{\varepsilon s}\left(q \mathrm{e}^{-(1-q) \varepsilon s}|x(q s)|^{\beta+2}-|x(s)|^{\beta+2}\right) \mathrm{d} s \\
& +\frac{\bar{b}}{q} \int_{0}^{t} \mathrm{e}^{\varepsilon s}\left(q \mathrm{e}^{-(1-q) \varepsilon s}|x(q s)|^{\gamma+2}-|x(s)|^{\gamma+2}\right) \mathrm{d} s \\
& -c_{0} \int_{0}^{t} \mathrm{e}^{\varepsilon s}|x(s)|^{2} \mathrm{~d} s .
\end{aligned}
$$

Making use of the property of the integral, we may estimate

$$
\begin{aligned}
\int_{0}^{t} \mathrm{e}^{\varepsilon s} & \left(q \mathrm{e}^{-(1-q) \varepsilon s}|x(q s)|^{\beta+2}-|x(s)|^{\beta+2}\right) \mathrm{d} s \\
& =\int_{0}^{t}\left(q \mathrm{e}^{\varepsilon q s}|x(q s)|^{\beta+2}-\mathrm{e}^{\varepsilon s}|x(s)|^{\beta+2}\right) \mathrm{d} s
\end{aligned}
$$

$$
\begin{aligned}
& \leq \int_{0}^{q t} \mathrm{e}^{\varepsilon s}|x(s)|^{\beta+2} \mathrm{~d} s-\int_{0}^{t} \mathrm{e}^{\varepsilon s}|x(s)|^{\beta+2} \mathrm{~d} s \\
& =-\int_{q t}^{t} \mathrm{e}^{\varepsilon s}|x(s)|^{\beta+2} \mathrm{~d} s .
\end{aligned}
$$

Similarly,

$$
\begin{gathered}
\int_{0}^{t} \mathrm{e}^{\varepsilon s}\left(q \mathrm{e}^{-(1-q) \varepsilon s}|x(q s)|^{\gamma+2}-|x(s)|^{\gamma+2}\right) \mathrm{d} s \\
\leq-\int_{q t}^{t} \mathrm{e}^{\varepsilon s}|x(s)|^{\gamma+2} \mathrm{~d} s .
\end{gathered}
$$

Substituting for (25) and (26) into (24) yields

$$
\begin{aligned}
\mathrm{e}^{\varepsilon t} V(x(t)) \leq & V(x(0))+M(t)-\frac{2 \bar{a}}{q} \int_{q t}^{t} \mathrm{e}^{\varepsilon s}|x(s)|^{\beta+2} \mathrm{~d} s \\
& -\frac{\bar{b}}{q} \int_{q t}^{t} \mathrm{e}^{\varepsilon s}|x(s)|^{\gamma+2} \mathrm{~d} s-\bar{c}_{0} \int_{0}^{t} \mathrm{e}^{\varepsilon s}|x(s)|^{2} \mathrm{~d} s .
\end{aligned}
$$

Applying the nonnegative semimartingale convergence theorem (see [33]) in (27), we have

$$
\lim \sup _{t \rightarrow \infty} e^{\varepsilon t} V(x(t))<\infty \text { a.s. }
$$

That is, there is a finite positive random variable $C_{0}$ such that $\sup _{0 \leq t<\infty} \mathrm{e}^{\varepsilon t} V(x(t)) \leq C_{0}$ a.s. This implies $\limsup _{t \rightarrow \infty}(1 / t) \log |x(t)| \leq-\varepsilon / 2$ a.s. The proof is complete.

\section{Stability of the Numerical Solution for Linear SPDE}

In the section, we will establish almost surely exponential stability of EM method numerical solution under the following linear growth conditions.

(H4) The Linear Growth Conditions. For any $x \in \mathbb{R}^{n}$, there exist positive constants $a, \bar{a}, c, \bar{c}, b, \bar{b}$ such that

$$
\begin{gathered}
\langle x(s), f(x(s), x(q s))\rangle \leq-a|x(s)|^{2}+\bar{a} \mathrm{e}^{-(1-q) \varepsilon s}|x(q s)|^{2} \\
|f(x(s), x(q s))|^{2} \leq c|x(s)|^{2}+\bar{c} \mathrm{e}^{-(1-q) \varepsilon s}|x(q s)|^{2} \\
\quad|g(x(s), x(q s))|^{2} \leq b|x(s)|^{2}+\bar{b} \mathrm{e}^{-(1-q) \varepsilon s}|x(q s)|^{2}
\end{gathered}
$$

Clearly, let $\alpha=\beta=\gamma=\widetilde{a}=\widetilde{b}=0$ in (H2) and (H3), which implies (H4). Similar to Theorems 2 and 3, we may obtain the following result.

Theorem 4. Let (H1) and (H4) hold with $2 a>(2 \bar{a}+\bar{b}+\bar{c}) / q+$ $b+c$. Then for any initial data $x_{0}$, there almost surely exists a unique global solution $x(t)$ to (5) on $t \geq 0$. Moreover, for 
$\varepsilon \leq 2 a-b-c-(2 \bar{a}+\bar{b}+\bar{c}) / q$, the solution is almost surely exponentially stable; that is,

$$
\lim \sup _{t \rightarrow \infty} \frac{1}{t} \log |x(t)| \leq-\frac{\varepsilon}{2} \quad \text { a.s. }
$$

Now we define the Euler-Maruyama approximate solution for (5). Given a step size $\Delta \in(0,1)$, compute the discrete approximations $X_{k} \approx x_{0}, t_{k}=k \Delta$ by setting $X_{0}=x_{0}$ and performing

$$
X_{k+1}=X_{k}+f\left(X_{k}, X_{[q k]}\right) \Delta+g\left(X_{k}, X_{[q k]}\right) \Delta w_{k},
$$

where the increments $\Delta w_{k}=w\left(t_{k+1}\right)-w\left(t_{k}\right), k=1, \ldots$, are independent $N(0, \Delta)$-distributed Gaussian random variables $\mathscr{F}_{t_{k}}$-measurable at the mesh-point $t_{k}$.

The following discrete semimartingale theorem plays an important role in the section.

Lemma 5 (see [32]). Let $\left\{A_{i}\right\}$ and $\left\{U_{i}\right\}$ be two nonnegative random variables such that both $A_{i}$ and $U_{i}$ are $\mathscr{F}_{i-1}{ }^{-}$ measurable for $i=1,2, \ldots$ with $A_{0}=U_{0}=0$ a.s. Let $\left\{M_{i}\right\}$ be a real-valued local martingale with $M_{0}=0$ a.s. Let $\zeta$ be a nonnegative $\mathscr{F}_{0}$-measurable random variable. Assume that $\left\{X_{i}\right\}$ is a nonnegative semimartingale with the Doob-Mayer decomposition $X_{i}=\zeta+A_{i}-U_{i}+M_{i}$. If $\lim _{t \rightarrow \infty} A_{i}<\infty$ a.s., then for almost all $\omega \in \Omega \lim _{t \rightarrow \infty} X_{i}<\infty, \lim _{t \rightarrow \infty} U_{i}<\infty$. That is, all of the three processes $X_{i}$ and $U_{i}$ converge to finite random variables.

Theorem 6. Let (H1) and (H4) hold with $2 a>(2 \bar{a}+m \bar{b}+$ $\bar{c}) / q+m b+c$. Then for $\varepsilon \leq 2 a-m b-c-(2 \bar{a}+m \bar{b}+\bar{c}) / q$, there exists a sufficiently small $\Delta^{*} \in(0,1)$ such that the approximate solution $\left\{X_{k}\right\}$ defined by (33) satisfies

$$
\lim \sup _{k \rightarrow \infty} \frac{1}{k \Delta} \log \left|X_{k}\right| \leq-\frac{\varepsilon}{2} \quad \text { a.s. }
$$

Proof. By (33) and (H4), we may compute

$$
\begin{aligned}
\left|X_{k+1}\right|^{2}= & \left\langle X_{k}+f\left(X_{k}, X_{[q k]}\right) \Delta+g\left(X_{k}, X_{[q k]}\right) \Delta w_{k},\right. \\
& \left.X_{k}+f\left(X_{k}, X_{[q k]}\right) \Delta+g\left(X_{k}, X_{[q k]}\right) \Delta w_{k}\right\rangle \\
= & \left|X_{k}\right|^{2}+2\left\langle X_{k}, f\left(X_{k}, X_{[q k]}\right)\right\rangle \Delta \\
& +\left|f\left(X_{k}, X_{[q k]}\right)\right|^{2} \Delta^{2}+\left|g\left(X_{k}, X_{[q k]}\right) \Delta w_{k}\right|^{2} \\
& +2\left\langle X_{k}+f\left(X_{k}, X_{[q k]}\right) \Delta, g\left(X_{k}, X_{[q k]}\right) \Delta w_{k}\right\rangle \\
= & \left|X_{k}\right|^{2}+2\left\langle X_{k}, f\left(X_{k}, X_{[q k]}\right)\right\rangle \Delta \\
& +\left(\left|f\left(X_{k}, X_{[q k]}\right)\right|^{2} \Delta+\left|g\left(X_{k}, X_{[q k]}\right)\right|^{2}\right) \Delta \\
& +\left|g\left(X_{k}, X_{[q k]}\right)\right|^{2}\left(\left.\Delta w_{k}\right|^{2}-m \Delta\right) \\
& +2\left\langle X_{k}+f\left(X_{k}, X_{[q k]}\right) \Delta, g\left(X_{k}, X_{[q k]}\right) \Delta w_{k}\right\rangle
\end{aligned}
$$

$$
\begin{aligned}
= & \left(1-2 a \Delta+c \Delta^{2}+m b \Delta\right)\left|X_{k}\right|^{2} \\
& +(2 \bar{a}+\bar{c} \Delta+m \bar{b}) \mathrm{e}^{-(1-q) \varepsilon k \Delta}\left|X_{[q k]}\right|^{2} \Delta \\
& +\left|g\left(X_{k}, X_{[q k]}\right)\right|^{2}\left(\left.\Delta w_{k}\right|^{2}-m \Delta\right) \\
& +2\left\langle X_{k}+f\left(X_{k}, X_{[q k]}\right) \Delta, g\left(X_{k}, X_{[q k]}\right) \Delta w_{k}\right\rangle \\
= & \left(1-2 a \Delta+c \Delta^{2}+m b \Delta\right)\left|X_{k}\right|^{2} \\
& +(2 \bar{a}+\bar{c} \Delta+m \bar{b}) \mathrm{e}^{-(1-q) \varepsilon k \Delta}\left|X_{[q k]}\right|^{2} \Delta+s_{k},
\end{aligned}
$$

where $s_{k}=\left|g\left(X_{k}, X_{[q k]}\right)\right|^{2}\left(\left|\Delta w_{k}\right|^{2}-m \Delta\right)+2\left\langle X_{k}+f\left(X_{k}\right.\right.$, $\left.\left.X_{[q k]}\right) \Delta, g\left(X_{k}, X_{[q k]}\right) \Delta w_{k}\right\rangle$. By (35), it is easy to obtain

$$
\begin{aligned}
\mathrm{e}^{\varepsilon(k+1) \Delta} & \left|X_{k+1}\right|^{2}-\mathrm{e}^{\varepsilon k \Delta}\left|X_{k}\right|^{2} \\
= & \left(1-2 a \Delta+c \Delta^{2}+m b \Delta-\mathrm{e}^{-\varepsilon \Delta}\right) \mathrm{e}^{\varepsilon(k+1) \Delta}\left|X_{k}\right|^{2} \\
& +(2 \bar{a}+\bar{c} \Delta+m \bar{b}) \mathrm{e}^{\varepsilon(k q+1) \Delta}\left|X_{[q k]}\right|^{2} \Delta+\mathrm{e}^{\varepsilon(k+1) \Delta} s_{k} .
\end{aligned}
$$

With the help of recursive method, it is not difficult to get

$$
\begin{aligned}
\mathrm{e}^{\varepsilon k \Delta} \mid & \left.X_{k}\right|^{2} \\
= & \left|X_{0}\right|^{2}+\left(1-2 a \Delta+c \Delta^{2}+m b \Delta-\mathrm{e}^{-\varepsilon \Delta}\right) \\
& \times \sum_{i=0}^{k-1} \mathrm{e}^{\varepsilon(i+1) \Delta}\left|X_{i}\right|^{2}+(2 \bar{a}+\bar{c} \Delta+m \bar{b}) \\
& \times \sum_{i=0}^{k-1} \mathrm{e}^{\varepsilon(i+1) \Delta}\left[\mathrm{e}^{-(1-q) \varepsilon i \Delta}\left|X_{[q i]}\right|^{2}-\mathrm{e}^{\varepsilon \Delta}\left|X_{i}\right|^{2}\right] \Delta \\
& +(2 \bar{a}+\bar{c} \Delta+m \bar{b}) \mathrm{e}^{\varepsilon \Delta} \sum_{i=0}^{k-1} \mathrm{e}^{\varepsilon(i+1) \Delta}\left|X_{i}\right|^{2} \Delta+\sum_{i=0}^{k-1} \mathrm{e}^{\varepsilon(i+1) \Delta} s_{i} \\
= & \left|X_{0}\right|^{2}-\left[2 a-c \Delta-m b-\frac{1-\mathrm{e}^{-\varepsilon \Delta}}{\Delta}\right. \\
& +\sum_{i=0}^{k-1} \mathrm{e}^{\varepsilon(i+1) \Delta} s_{i} . \\
& +\sum_{i=0}^{k-1} \mathrm{e}^{\varepsilon(i+1) \Delta}\left(\mathrm{e}^{-(1-q) \varepsilon i \Delta}\left|X_{[q i]}\right|^{2}-\mathrm{e}^{\varepsilon \Delta}\left|X_{i}\right|^{2}\right) \Delta \\
& \times \sum_{i=0}^{k-1} \mathrm{e}^{\varepsilon(i+1) \Delta}\left|X_{i}\right|^{2} \Delta \\
& \left.-(2 \bar{a}+\bar{c} \Delta+m \bar{b}) \mathrm{e}^{\varepsilon \Delta}\right]
\end{aligned}
$$


Obviously, $\sum_{i=0}^{k-1} \mathrm{e}^{\varepsilon(i+1) \Delta} s_{i}$ is a martingale. Let [qi] $=j$; then $j \leq q i<j+1$, so $q i-1<j \leq q i$. If $0 \leq i \leq k-1$, then $-1<j \leq q(k-1) \leq[q k]+1-q \leq[q k]+1$. This implies that

$$
\begin{aligned}
& \sum_{i=0}^{k-1} \mathrm{e}^{\varepsilon(i+1) \Delta}\left[\mathrm{e}^{-(1-q) \varepsilon i \Delta}\left|X_{[q i]}\right|^{2}-\mathrm{e}^{\varepsilon \Delta}\left|X_{i}\right|^{2}\right] \Delta \\
& \quad=\sum_{i=0}^{k-1} \mathrm{e}^{\varepsilon(q i+1) \Delta}\left|X_{[q i]}\right|^{2}-\sum_{i=0}^{k-1} \mathrm{e}^{\varepsilon(i+2) \Delta}\left|X_{i}\right|^{2} \\
& \leq \sum_{i=0}^{[q k]+1} \mathrm{e}^{\varepsilon(i+2) \Delta}\left|X_{i}\right|^{2}-\sum_{i=0}^{k-1} \mathrm{e}^{\varepsilon(i+2) \Delta}|X(i \Delta)|^{\alpha+2} \\
& \leq-\sum_{i=[q k]+2}^{k-1} \mathrm{e}^{\varepsilon(i+2) \Delta}\left|X_{i}\right|^{2} .
\end{aligned}
$$

Denote by

$$
f(\Delta)=2 a-c \Delta-m b+\frac{\mathrm{e}^{-\varepsilon \Delta}-1}{\Delta}-\mathrm{e}^{\varepsilon \Delta}(2 \bar{a}+\bar{c} \Delta+m \bar{b}) .
$$

By the Taylor series, we have

$$
\begin{gathered}
\mathrm{e}^{-\varepsilon \Delta}=1-\varepsilon \Delta+\frac{(\varepsilon \Delta)^{2}}{2}-\frac{(\varepsilon \Delta)^{3}}{3 !}+o(\varepsilon \Delta)>1-\varepsilon \Delta, \\
\frac{\mathrm{e}^{-\varepsilon \Delta}-1}{\Delta}>-\varepsilon
\end{gathered}
$$

Therefore $f(\Delta)>2 a-c \Delta-m b-\varepsilon-e^{\varepsilon \Delta}(2 \bar{a}+\bar{c} \Delta+m \bar{b})$. Noting that $2 a>(2 \bar{a}+m \bar{b}+\bar{c}) / q+m b+c, q \in(0,1)$, for any given $\varepsilon$, choose a sufficiently small $\Delta^{*}\left(\Delta^{*}<\bar{\Delta}\right)$ such that for all $\Delta<\Delta^{*}$,

$$
2 a-c \Delta-m b-\varepsilon-\mathrm{e}^{\varepsilon \Delta}(2 \bar{a}+\bar{c} \Delta+m \bar{b})>0 .
$$

Substituting for (38) and (41) into (37), Lemma 5 implies that there exists a positive constant $C_{0}$ such that $\mathrm{e}^{\varepsilon k \Delta}\left|X_{k}\right|^{2} \leq C_{0}$. That is, $\lim \sup _{k \rightarrow \infty}(1 / k \Delta) \log \left|X_{k}\right| \leq-\varepsilon / 2$ a.s. The proof is complete.

\section{Stability of Numerical Solution for Highly Nonlinear SPDE}

In the section, we will prove that the backward EM method can preserve the almost surely exponential stability of the true solution of highly nonlinear stochastic pantograph differential equation.

(H5) The Polynomial Growth Conditions. For any $x \in \mathbb{R}^{d}$, there exist positive constants $\alpha, a, \bar{a}, \widetilde{a}, b, \bar{b}, \widetilde{b}$ such that

$$
\begin{aligned}
& \langle x(s), f(x(s), x(q s))\rangle \\
& \quad \leq-a|x(s)|^{\alpha+2}+\bar{a} \mathrm{e}^{-(1-q) \varepsilon s}|x(q s)|^{\alpha+2}-\tilde{a}|x(s)|^{2}, \\
& |g(x(s), x(q s))|^{2} \\
& \quad \leq b|x(s)|^{\alpha+2}+\bar{b} \mathrm{e}^{-(1-q) \varepsilon s}|x(q s)|^{\alpha+2}+\tilde{b}|x(s)|^{2} .
\end{aligned}
$$

By Theorems 2 and 3, (5) has a unique global solution and the solution is almost surely exponentially stable.

Given a step size $\Delta \in(0,1)$, and for $t \in[0, T], M \Delta=T$ for some positive integer $M$. Let $t_{k}=k \Delta(k \geq 0),[t / \Delta]$ be the integer part of $t / \Delta$. Define the backward Euler-Maruyama method as follows:

$$
X_{k+1}=X_{k}+f\left(X_{k+1}, X_{[q(k+1)]}\right) \Delta+g\left(X_{k}, X_{[q k]}\right) \Delta w\left(t_{k}\right) \text {. }
$$

To guarantee that this method is well defined, we impose the following one-sided Lipschitz condition on the drift coefficient $f(x, y)$.

(H6) One-Sided Lipschitz Condition. There exists a positive constant $\lambda>0$ such that for any $x_{i} \in \mathbb{R}^{n}(i=1,2)$

$$
\left\langle x_{1}-x_{2}, f\left(x_{1}, y\right)-f\left(x_{2}, y\right)\right\rangle \leq \lambda\left|x_{1}-x_{2}\right|^{2} .
$$

Applying a fixed point theorem one can prove that (44) has a unique solution $X_{k+1}$; given $X_{k}$ if $\lambda \Delta<1$, then the backward EM scheme (44) is well defined (see, e.g., [28]). From now on we always assume that $\Delta<\lambda^{-1}$.

Theorem 7. Let (H1), (H5) and (H6) hold with $2 \widetilde{a}>m \tilde{b}, 2 a>$ $m b+(2 \bar{a}+m \bar{b}) / q$; there exists a sufficiently small $\Delta^{*} \in$ $(0,1)$ such that the approximate solution $\left\{X_{k}\right\}$ defined by $(44)$ satisfies

$$
\lim \sup _{k \rightarrow \infty} \frac{1}{k \Delta} \log \left|X_{k}\right| \leq-\frac{\varepsilon}{2} \quad \text { a.s., }
$$

where $\varepsilon<2 \widetilde{a}-m \tilde{b}$.

Proof. By (44) and (H5), we may compute

$$
\begin{aligned}
\left|X_{k+1}\right|^{2}= & \left\langle X_{k+1}, X_{k}+f\left(X_{k+1}, X_{[q(k+1)]}\right) \Delta\right. \\
& \left.+g\left(X_{k}, X_{[q k]}\right) \Delta w_{k}\right\rangle \\
= & \left\langle X_{k+1}, f\left(X_{k+1}, X_{[q(k+1)]}\right)\right\rangle \Delta \\
& +\left\langle X_{k+1}, X_{k}+g\left(X_{k}, X_{[q k]}\right) \Delta w_{k}\right\rangle \\
\leq & -a\left|X_{k+1}\right|^{\alpha+2} \Delta+\bar{a} \mathrm{e}^{-\varepsilon(1-q)(k+1) \Delta}\left|X_{[q(k+1)]}\right|^{\alpha+2} \Delta \\
& -\tilde{a}\left|X_{k+1}\right|^{2} \Delta+\frac{1}{2}\left|X_{k+1}\right|^{2}+\frac{1}{2}\left|X_{k}\right|^{2} \\
& +\frac{1}{2}\left|g\left(X_{k}, X_{[q k]}\right)\right|^{2}\left|\Delta w_{k}\right|^{2} \\
& +\left\langle X_{k}, g\left(X_{k}, X_{[q k]}\right)\right\rangle \Delta w_{k}
\end{aligned}
$$




$$
\begin{aligned}
= & \left(\frac{1}{2}-\tilde{a} \Delta\right)\left|X_{k+1}\right|^{2}+\bar{a} \mathrm{e}^{-\varepsilon(1-q)(k+1) \Delta}\left|X_{[q(k+1)]}\right|^{\alpha+2} \Delta \\
& -a\left|X_{k+1}\right|^{\alpha+2} \Delta+\frac{1}{2}\left|X_{k}\right|^{2} \\
& +\frac{1}{2}\left(b\left|X_{k}\right|^{\alpha+2}+\bar{b} \mathrm{e}^{-\varepsilon(1-q) k \Delta}\left|X_{[q k]}\right|^{\alpha+2}+\widetilde{b}\left|X_{k}\right|^{2}\right) \\
& \times\left|\Delta w_{k}\right|^{2}+\left\langle X_{k}, g\left(X_{k}, X_{[q k]}\right)\right\rangle \Delta w_{k} .
\end{aligned}
$$

That is,

$$
\begin{aligned}
& (1+2 \widetilde{a} \Delta)\left|X_{k+1}\right|^{2} \\
= & (1+m \widetilde{b} \Delta)\left|X_{k}\right|^{2}+2 \bar{a} \mathrm{e}^{-\varepsilon(1-q)(k+1) \Delta}\left|X_{[q(k+1)]}\right|^{\alpha+2} \Delta \\
& -2 a\left|X_{k+1}\right|^{\alpha+2} \Delta+\left(m b\left|X_{k}\right|^{\alpha+2}+m \bar{b} \mathrm{e}^{-\varepsilon(1-q) k \Delta}\left|X_{[q k]}\right|^{\alpha+2}\right) \\
& \times \Delta+s_{k}^{\Delta},
\end{aligned}
$$

where

$$
\begin{aligned}
s_{k}^{\Delta}= & \left(b\left|X_{k}\right|^{\alpha+2}+\bar{b} \mathrm{e}^{-\varepsilon(1-q) k \Delta}\left|X_{[q k]}\right|^{\alpha+2}+\widetilde{b}\left|X_{k}\right|^{2}\right) \\
& \times\left(\left|\Delta w_{k}\right|^{2}-m \Delta\right)+2\left\langle X_{k}, g\left(X_{k}, X_{[q k]}\right)\right\rangle \Delta w_{k} .
\end{aligned}
$$

According to (48), we may obtain

$$
\begin{aligned}
(1+2 \widetilde{a} \Delta) & {\left[\mathrm{e}^{\varepsilon(k+1) \Delta}\left|X_{k+1}\right|^{2}-\mathrm{e}^{\varepsilon k \Delta}\left|X_{k}\right|^{2}\right] } \\
= & {\left[1+m \widetilde{b} \Delta-(1+2 \widetilde{a} \Delta) \mathrm{e}^{-\varepsilon \Delta}\right] \mathrm{e}^{\varepsilon(k+1) \Delta}\left|X_{k}\right|^{2} } \\
& +2 \bar{a} \mathrm{e}^{\varepsilon q(k+1) \Delta}\left|X_{[q(k+1)]}\right|^{\alpha+2} \Delta-2 a \mathrm{e}^{\varepsilon(k+1) \Delta}\left|X_{k+1}\right|^{\alpha+2} \Delta \\
& +m b \mathrm{e}^{\varepsilon(k+1) \Delta}\left|X_{k}\right|^{\alpha+2} \Delta+m \bar{b} \mathrm{e}^{-\varepsilon(q k+1) \Delta}\left|X_{[q k]}\right|^{\alpha+2} \Delta \\
& +\mathrm{e}^{\varepsilon(k+1) \Delta} s_{k}^{\Delta} .
\end{aligned}
$$

With the help of recursive method, compute

$$
\begin{aligned}
(1+2 \tilde{a} \Delta) & \mathrm{e}^{\varepsilon k \Delta}\left|X_{k}\right|^{2} \\
= & (1+2 \widetilde{a} \Delta)\left|X_{0}\right|^{2}+\left[1+m \widetilde{b} \Delta-(1+2 \widetilde{a} \Delta) \mathrm{e}^{-\varepsilon \Delta}\right] \\
& \times \sum_{i=0}^{k-1} \mathrm{e}^{\varepsilon(i+1) \Delta}\left|X_{i}\right|^{2}-2 a \sum_{i=0}^{k-1} \mathrm{e}^{\varepsilon(i+1) \Delta}\left|X_{i+1}\right|^{\alpha+2} \Delta \\
& +2 \bar{a} \sum_{i=0}^{k-1} \mathrm{e}^{\varepsilon q(i+1) \Delta}\left|X_{[q(i+1)]}\right|^{\alpha+2} \Delta \\
& +m b \sum_{i=0}^{k-1} \mathrm{e}^{\varepsilon(i+1) \Delta}\left|X_{i}\right|^{\alpha+2} \Delta \\
& +m \bar{b} \sum_{i=0}^{k-1} \mathrm{e}^{\varepsilon(q i+1) \Delta}\left|X_{[q i]}\right|^{\alpha+2} \Delta+\sum_{i=0}^{k-1} \mathrm{e}^{\varepsilon(i+1) \Delta} s_{i}^{\Delta}
\end{aligned}
$$

$$
\begin{aligned}
& =(1+2 \widetilde{a} \Delta)\left|X_{0}\right|^{2}-\left[1+2 \widetilde{a} \Delta-(1+\bar{m} \tilde{b} \Delta) \mathrm{e}^{\varepsilon \Delta}\right] \\
& +\sum_{i=0}^{k-1} \mathrm{e}^{\varepsilon i \Delta}\left|X_{i}\right|^{2}-2 a \sum_{i=1}^{k} \mathrm{e}^{\varepsilon i \Delta}\left|X_{i}\right|^{\alpha+2} \Delta \\
& +m b \sum_{i=0}^{k-1} \mathrm{e}^{\varepsilon(i+1) \Delta}\left|X_{i}\right|^{\alpha+2} \Delta+2 \bar{a} \sum_{i=0}^{k-1} \mathrm{e}^{\varepsilon(i+1) \Delta}\left|X_{i}\right|^{\alpha+2} \Delta \\
& +m \bar{b} \sum_{i=0}^{k-1} \mathrm{e}^{\varepsilon(i+2) \Delta}\left|X_{i}\right|^{\alpha+2} \Delta \\
& +2 \bar{a} \sum_{i=0}^{k-1} \mathrm{e}^{\varepsilon(i+1) \Delta}\left(\mathrm{e}^{-\varepsilon(1-q)(i+1) \Delta}\left|X_{[q(i+1)]}\right|^{\alpha+2}\right. \\
& +m \bar{b} \sum_{i=0}^{k-1} \mathrm{e}^{\varepsilon(i+1) \Delta}\left(\mathrm{e}^{-\varepsilon(1-q) i \Delta}\left|X_{[q i]}\right|^{\alpha+2}-\mathrm{e}^{\varepsilon \Delta}\left|X_{i}\right|^{\alpha+2}\right) \Delta \\
& +S_{k},
\end{aligned}
$$

where $S_{k}=\sum_{i=0}^{k-1} \mathrm{e}^{\varepsilon(i+1) \Delta} s_{k}^{\Delta}$. Obviously, $S_{k}$ is a martingale. Let [qi] $=j$; then $j \leq q i<j+1$, so $q i-1<j \leq q i$. If $0 \leq i \leq k-1$; then $-1<j \leq q(k-1) \leq[q k]+1-q \leq[q k]+1$. This implies

$$
\begin{aligned}
& \sum_{i=0}^{k-1} \mathrm{e}^{\varepsilon(i+1) \Delta}\left(\mathrm{e}^{-\varepsilon(1-q) i \Delta}\left|X_{[q i]}\right|^{\alpha+2}-\mathrm{e}^{\varepsilon \Delta}\left|X_{i}\right|^{\alpha+2}\right) \\
& =\sum_{i=0}^{k-1} \mathrm{e}^{\varepsilon(1+q i) \Delta}\left|X_{[q i]}\right|^{\alpha+2}-\sum_{i=0}^{k-1} \mathrm{e}^{\varepsilon(i+2) \Delta}\left|X_{i}\right|^{\alpha+2} \\
& =\sum_{i=0}^{[q k]+1} \mathrm{e}^{\varepsilon(i+2) \Delta}\left|X_{i}\right|^{\alpha+2}-\sum_{i=0}^{k-1} \mathrm{e}^{\varepsilon(i+2) \Delta}\left|X_{i}\right|^{\alpha+2} \\
& \leq-\sum_{i=[q k]+2}^{k-1} \mathrm{e}^{\varepsilon(i+2) \Delta}\left|X_{i}\right|^{\alpha+2} .
\end{aligned}
$$

Similarly,

$$
\begin{aligned}
& \left.\left.\sum_{i=0}^{k-1} \mathrm{e}^{-\varepsilon(i+1) \Delta}\left[\mathrm{e}^{\varepsilon(1-q)(i+1) \Delta} \mid X_{[q(i+1)]}\right)\right|^{\alpha+2}-\left|X_{i}\right|^{\alpha+2}\right) \\
& \leq-\sum_{i=[q k]+2}^{k-1} \mathrm{e}^{\varepsilon(i+1) \Delta}\left|X_{i}\right|^{\alpha+2} .
\end{aligned}
$$

Substituting for (52) and (53) into (51) yields

$$
\begin{aligned}
(1+ & 2 \widetilde{a} \Delta) \mathrm{e}^{\varepsilon k \Delta}\left|X_{k}\right|^{2} \\
= & (1+2 \widetilde{a} \Delta)\left|X_{0}\right|^{2}-\left[1+2 \tilde{a} \Delta-(1+m \tilde{b} \Delta) \mathrm{e}^{\varepsilon \Delta}\right] \\
& \times \sum_{i=0}^{k-1} \mathrm{e}^{\varepsilon i \Delta}\left|X_{i}\right|^{2}+\left(-2 a+m b \mathrm{e}^{\varepsilon \Delta}+2 \bar{a} \mathrm{e}^{\varepsilon \Delta}+m \bar{b} \mathrm{e}^{2 \varepsilon \Delta}\right)
\end{aligned}
$$




$$
\begin{aligned}
& \times \sum_{i=1}^{k-1} \mathrm{e}^{\varepsilon i \Delta}\left|X_{i}\right|^{\alpha+2} \Delta-2 a \mathrm{e}^{\varepsilon k \Delta}\left|X_{k}\right|^{\alpha+2} \Delta \\
& +\left(2 \bar{a}+m b+m \bar{b} \mathrm{e}^{\varepsilon \Delta}\right) \mathrm{e}^{\varepsilon \Delta}\left|X_{0}\right|^{\alpha+2} \Delta \\
& -2 \bar{a} \sum_{i=[q k]+2}^{k-1} \mathrm{e}^{\varepsilon(i+1) \Delta}\left|X_{i}\right|^{\alpha+2} \Delta \\
& -m \bar{b} \sum_{i=[q k]+2}^{k-1} \mathrm{e}^{\varepsilon(i+2) \Delta}\left|X_{i}\right|^{\alpha+2} \Delta+S_{k} .
\end{aligned}
$$

Denote by $f(\Delta)=1+2 \widetilde{a} \Delta-(1+m \widetilde{b} \Delta) \mathrm{e}^{\varepsilon \Delta}$. Differentiating with respect to $\Delta$ yields

$$
\begin{aligned}
& f^{\prime}(\Delta)=2 \widetilde{a}-m \tilde{b} e^{\varepsilon \Delta}-(1+m \tilde{b} \Delta) \varepsilon e^{\varepsilon \Delta}, \\
& f^{\prime \prime}(\Delta)=-2 m \tilde{b} \varepsilon e^{\varepsilon \Delta}-(1+m \widetilde{b} \Delta) \varepsilon^{2} e^{\varepsilon \Delta} .
\end{aligned}
$$

Clearly, $f^{\prime}(0)=2 \widetilde{a}-m \widetilde{b}-\varepsilon>0, f^{\prime \prime}(0)<0$; then there exists a $\bar{\Delta}>0$ such that $f^{\prime}(\bar{\Delta})=0 . f(\Delta)$ is an increase function for $\Delta<\bar{\Delta}$ and noting that $f(0)=0$ therefore there exists a sufficiently small $\Delta^{*}\left(\Delta^{*}<\bar{\Delta}\right)$ such that for all $\Delta<\Delta^{*}$,

$$
1+2 \widetilde{a} \Delta-(1+m \widetilde{b} \Delta) \mathrm{e}^{\varepsilon \Delta}>0 .
$$

On the other hand, since $2 \widetilde{a}>(2 \bar{a}+m \bar{b}) / q+b, q \in(0,1)$, then $2 a>2 \bar{a}+m \bar{b}+m \tilde{b}$, and there exists sufficiently small $\Delta<\Delta^{*}$ such that

$$
2 a-2 \bar{a} e^{\varepsilon \Delta}-m\left(b+\bar{b} e^{\varepsilon \Delta}\right) \mathrm{e}^{\varepsilon \Delta}>0 .
$$

Substituting for (56) and (57) into (54), Lemma 5 implies that there exists a positive constant $C_{0}$ such that

$$
(1+2 a \Delta) \mathrm{e}^{\varepsilon k \Delta}\left|X_{k}\right|^{2} \leq C_{0}
$$

That is,

$$
\lim \sup _{k \rightarrow \infty} \frac{1}{k \Delta} \log \left|X_{k}\right| \leq-\frac{\varepsilon}{2} \quad \text { a.s. }
$$

The proof is complete.

Example 8. Consider the following nonlinear scalar SPDE

$$
\begin{aligned}
\mathrm{d} x(t)= & {\left[-0.5 x(t)-5 x^{5}(t)+3 \mathrm{e}^{-0.25 \varepsilon t} x^{5}(q t)\right] \mathrm{d} t } \\
& +x^{3}(t) \mathrm{d} w(t),
\end{aligned}
$$

where $w(t)$ is scalar Brownian motion. Define $f(x, y, t)=$ $-0.5 x-5 x^{5}+3 \mathrm{e}^{-0.25 s t} y^{5}, g(x, y, t)=x^{3}$. Compute

$$
\begin{aligned}
& f\left(x_{1}, y, t\right)-f\left(x_{2}, y, t\right) \\
& \leq-0.5\left(x_{1}-x_{2}\right)-5\left(x_{1}^{5}-x_{2}^{5}\right) \\
& \leq-0.5\left(x_{1}-x_{2}\right)\left[1+10\left(x_{1}^{4}+x_{1}^{3} x_{2}+x_{2}^{2} x_{2}^{2}\right.\right. \\
& \left.\left.+x_{1} x_{2}^{3}+x_{2}^{4}\right)\right] .
\end{aligned}
$$

Noting that $a^{2}+b^{2} \geq(a+b)^{2} / 2$, compute

$$
\begin{aligned}
x_{1}^{4}+ & x_{1}^{3} x_{2}+x_{2}^{2} x_{2}^{2}+x_{1} x_{2}^{3}+x_{2}^{4} \\
& \geq \frac{\left(x_{1}^{2}+x_{2}^{2}\right)^{2}}{2}+x_{1} x_{2}\left(x_{1}^{2}+x_{2}^{2}\right)+\left(x_{1} x_{2}\right)^{2} \\
& \geq\left[\frac{x_{1}^{2}+x_{2}^{2}}{2}+x_{1} x_{2}\right]^{2},
\end{aligned}
$$

which implies

$$
\left\langle x_{1}-x_{2}, f\left(x_{1}, y, t\right)-f\left(x_{2}, y, t\right)\right\rangle \leq-0.5\left(x_{1}-x_{2}\right)^{2} .
$$

That implies that $f(x, y, t)$ satisfies the one-sided growth condition. By using the inequality $a^{p} b^{q} \leq(p /(p+q)) a^{p+q}+$ $(q /(p+q)) b^{p+q}$, it is easy to compute

$$
\begin{aligned}
\langle x, f(x, y, t)\rangle & \leq-0.5 x^{2}-5 x^{6}+3 e^{-0.25 \varepsilon t} y^{5} x \\
& \leq-0.5 x^{2}-4.5 x^{6}+2.5 y^{6}
\end{aligned}
$$

and $|g(x(t), y(t))|^{2} \leq x^{6}$. By Theorems 2 and $7,(60)$ has unique global solution and the solution is almost surely exponentially stable.

\section{Conflict of Interests}

The author declares that there is no conflict of interests regarding the publication of this paper.

\section{Acknowledgments}

The author expresses their sincere gratitude to two anonymous referees for their detailed comments and helpful suggestions. This project is supported by NNSF (11301198) and Fundamental Research Funds for the Central Universities (2011QN167).

\section{References}

[1] Y. Hu and F. Wu, "Stochastic Kolmogorov-type population dynamics with infinite distributed delays," Acta Applicandae Mathematicae, vol. 110, no. 3, pp. 1407-1428, 2010.

[2] F. Wei and K. Wang, “The existence and uniqueness of the solution for stochastic functional differential equations with infinite delay," Journal of Mathematical Analysis and Applications, vol. 331, no. 1, pp. 516-531, 2007.

[3] S. Zhou, Z. Wang, and D. Feng, "Stochastic functional differential equations with infinite delay," Journal of Mathematical Analysis and Applications, vol. 357, no. 2, pp. 416-426, 2009.

[4] S. Zhou, S. Hu, and L. Cen, "Stochastic Kolmogorov-type system with infinite delay," Applied Mathematics and Computation, vol. 218, no. 2, pp. 207-218, 2011.

[5] C. Huang and J. Cao, "Almost sure exponential stability of stochastic cellular neural networks with unbounded distributed delays," Neurocomputing, vol. 72, no. 13-15, pp. 3352-3356, 2009.

[6] X. Li and X. Fu, "Stability analysis of stochastic functional differential equations with infinite delay and its application to recurrent neural networks," Journal of Computational and Applied Mathematics, vol. 234, no. 2, pp. 407-417, 2010. 
[7] X. Meng, S. Hu, and P. Wu, "Pathwise estimation of stochastic differential equations with unbounded delay and its application to stochastic pantograph equations," Acta Applicandae Mathematicae, vol. 113, no. 2, pp. 231-246, 2011.

[8] X. Meng, M. Tian, and S. Hu, "Stability analysis of stochastic recurrent neural networks with unbounded time-varying delays," Neurocomputing, vol. 74, no. 6, pp. 949-953, 2011.

[9] J. R. Ockendon and A. B. Tayler, "The dynamics of a current collection system for an electric locomotive," Proceedings of the Royal Society A, vol. 322, pp. 447-468, 1971.

[10] J. A. D. Appleby, "Decay and growth rates of solutions of scalar stochastic delay differential equations with unbounded delay and state-dependent noise," Stochastics and Dynamics, vol. 5, no. 2, pp. 133-147, 2005.

[11] C. T. H. Baker and E. Buckwar, "Continuous $\theta$-methods for the stochastic pantograph equation," Electronic Transactions on Numerical Analysis, vol. 11, pp. 131-151, 2000.

[12] P. Balasubramaniam and S. K. Ntouyas, "Controllability for neutral stochastic functional differential inclusions with infinite delay in abstract space," Journal of Mathematical Analysis and Applications, vol. 324, no. 1, pp. 161-176, 2006.

[13] Z. Fan, M. Liu, and W. Cao, "Existence and uniqueness of the solutions and convergence of semi-implicit Euler methods for stochastic pantograph equations," Journal of Mathematical Analysis and Applications, vol. 325, no. 2, pp. 1142-1159, 2007.

[14] Z. Fan, M. Song, and M. Liu, "The $\alpha$ th moment stability for the stochastic pantograph equation," Journal of Computational and Applied Mathematics, vol. 233, no. 2, pp. 109-120, 2009.

[15] D. J. Higham, X. Mao, and C. Yuan, "Almost sure and moment exponential stability in the numerical simulation of stochastic differential equations," SIAM Journal on Numerical Analysis, vol. 45, no. 2, pp. 592-609, 2007.

[16] R. Li, M. Liu, and P. Wang, "Convergence of numerical solutions to stochastic pantograph equations with Markovian switching," Applied Mathematics and Computation, vol. 215, no. 1, pp. 414422, 2009.

[17] X. Mao, "Exponential stability of equidistant Euler-Maruyama approximations of stochastic differential delay equations," Journal of Computational and Applied Mathematics, vol. 200, no. 1, pp. 297-316, 2007.

[18] M. Milošević and M. Jovanović, "A Taylor polynomial approach in approximations of solution to pantograph stochastic differential equations with Markovian switching," Mathematical and Computer Modelling, vol. 53, no. 1-2, pp. 280-293, 2011.

[19] S. Zhou and F. Wu, "Convergence of numerical solutions to neutral stochastic delay differential equations with Markovian switching," Journal of Computational and Applied Mathematics, vol. 229, no. 1, pp. 85-96, 2009.

[20] X. Mao, "Numerical solutions of stochastic differential delay equations under the generalized Khasminskii-type conditions," Applied Mathematics and Computation, vol. 217, no. 12, pp. 55125524, 2011.

[21] M. Milošević, "Highly nonlinear neutral stochastic differential equations with time-dependent delay and the Euler-Maruyama method," Mathematical and Computer Modelling, vol. 54, no. 910, pp. 2235-2251, 2011.

[22] S. Zhou and Z. Fang, "Numerical approximation of nonlinear neutral stochastic functional differential equations," Journal of Applied Mathematics and Computing, vol. 41, no. 1-2, pp. 427445, 2013.
[23] S. Zhou and M. Xue, "Exponential stability for nonlinear hybrid stochastic pantograph equations and numerical approximation," Acta Mathematisa Scientia, vol. 4, pp. 1-17, 2014.

[24] D. J. Higham, X. Mao, and A. M. Stuart, "Exponential meansquare stability of numerical solutions to stochastic differential equations," LMS Journal of Computation and Mathematics, vol. 6, pp. 297-313, 2003.

[25] D. J. Higham, "Mean-square and asymptotic stability of the stochastic theta method," SIAM Journal on Numerical Analysis, vol. 38, no. 3, pp. 753-769, 2000.

[26] D. J. Higham, X. Mao, and C. Yuan, "Preserving exponential mean-square stability in the simulation of hybrid stochastic differential equations," Numerische Mathematik, vol. 108, no. 2, pp. 295-325, 2007.

[27] S. Pang, F. Deng, and X. Mao, "Almost sure and moment exponential stability of Euler-Maruyama discretizations for hybrid stochastic differential equations," Journal of Computational and Applied Mathematics, vol. 213, no. 1, pp. 127-141, 2008.

[28] X. Mao, Y. Shen, and A. Gray, "Almost sure exponential stability of backward Euler-Maruyama discretizations for hybrid stochastic differential equations," Journal of Computational and Applied Mathematics, vol. 235, no. 5, pp. 1213-1226, 2011.

[29] X. Mao and L. Szpruch, "Strong convergence and stability of implicit numerical methods for stochastic differential equations with non-globally Lipschitz continuous coefficients," Journal of Computational and Applied Mathematics, vol. 238, pp. 14-28, 2013.

[30] B. Liu and D. J. Hill, "Input-to-state stability for discrete timedelay systems via the Razumikhin technique," Systems \& Control Letters, vol. 58, no. 8, pp. 567-575, 2009.

[31] M. Wu, Y. He, and J.-H. She, "New delay-dependent stability criteria and stabilizing method for neutral systems," IEEE Transactions on Automatic Control, vol. 49, no. 12, pp. 22662271, 2004.

[32] F. Wu, X. Mao, and L. Szpruch, "Almost sure exponential stability of numerical solutions for stochastic delay differential equations," Numerische Mathematik, vol. 115, no. 4, pp. 681-697, 2010.

[33] S. Zhou, S. Xie, and Z. Fang, "Almost surely exponential stability of the backward Euler-Maruyama discretization for highly nonlinear stochastic functional differential equation," Applied Mathematics and Computation, vol. 236, no. 6, pp. 150-160, 2014. 


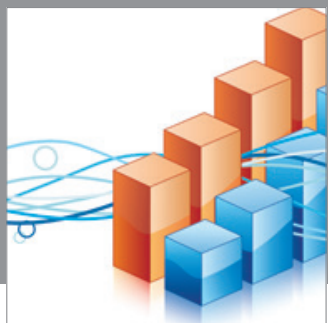

Advances in

Operations Research

mansans

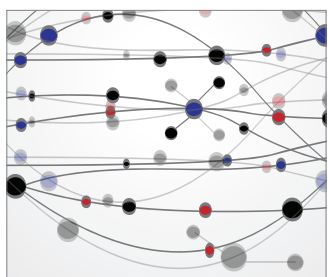

The Scientific World Journal
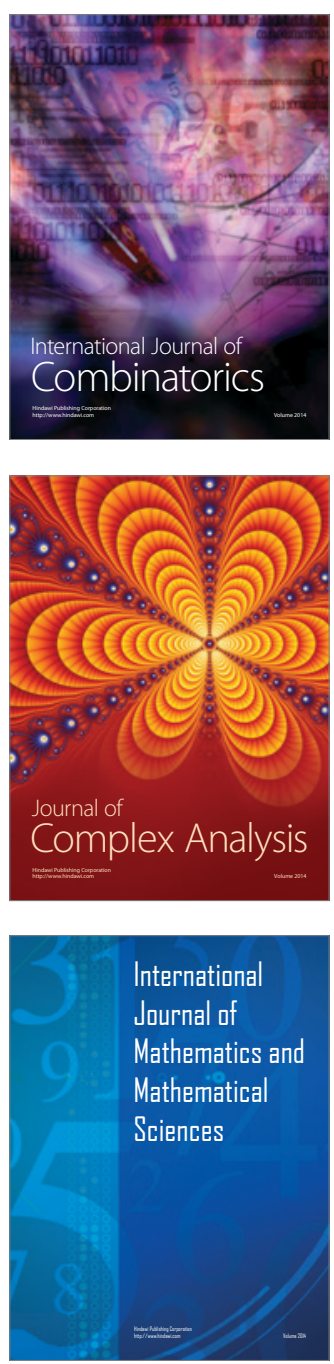
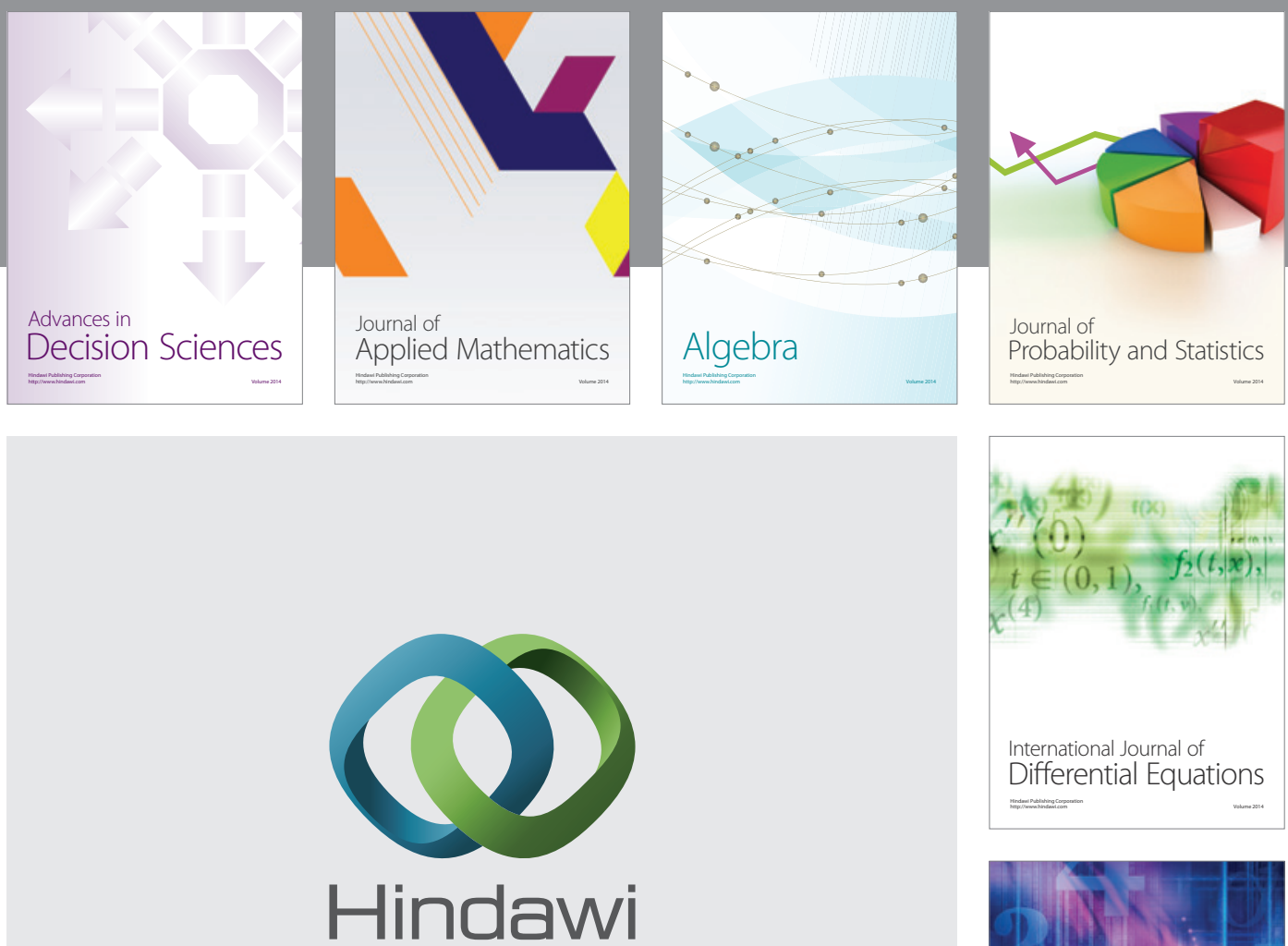

Submit your manuscripts at http://www.hindawi.com
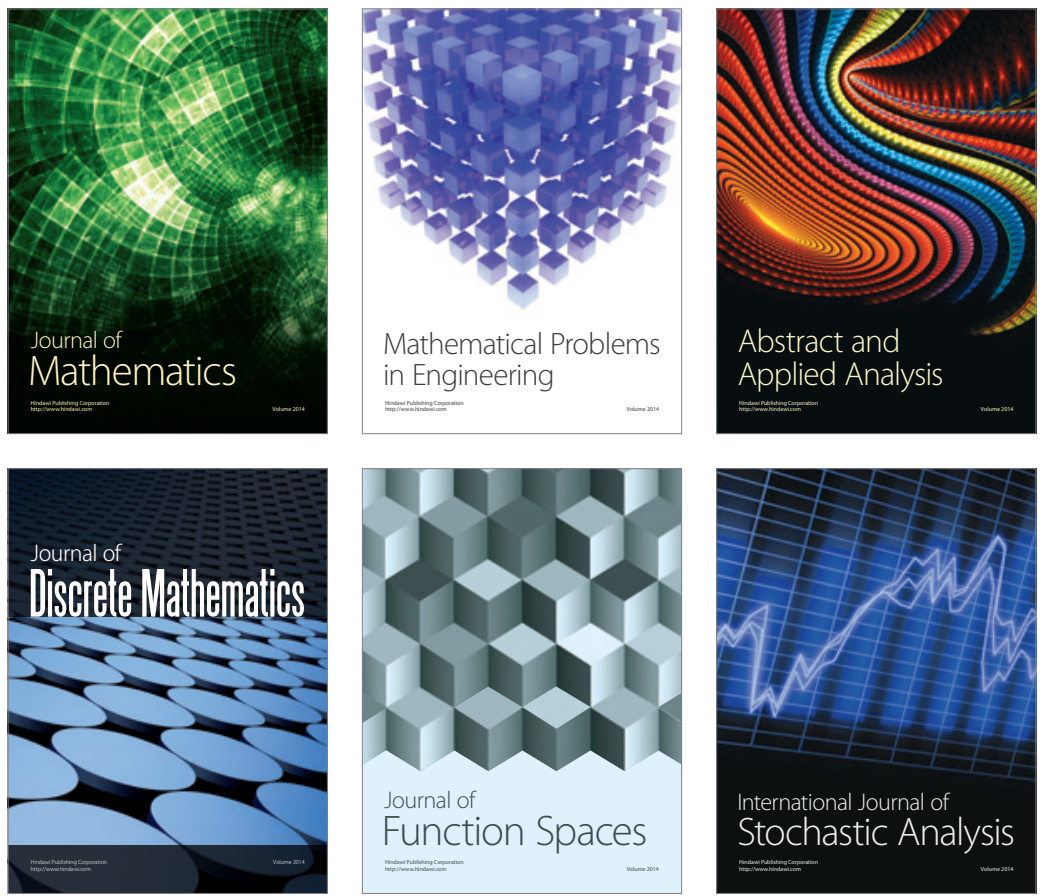

Journal of

Function Spaces

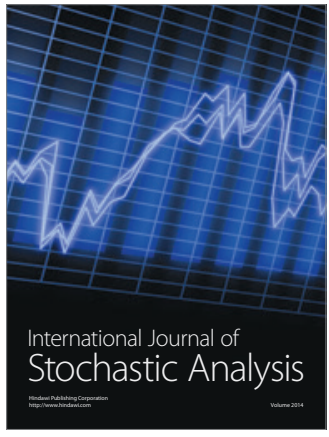

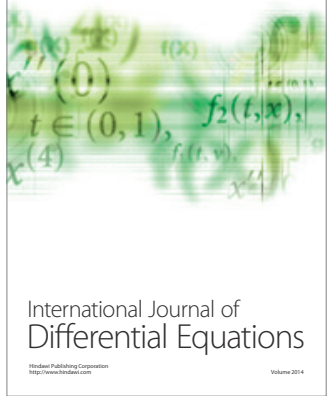
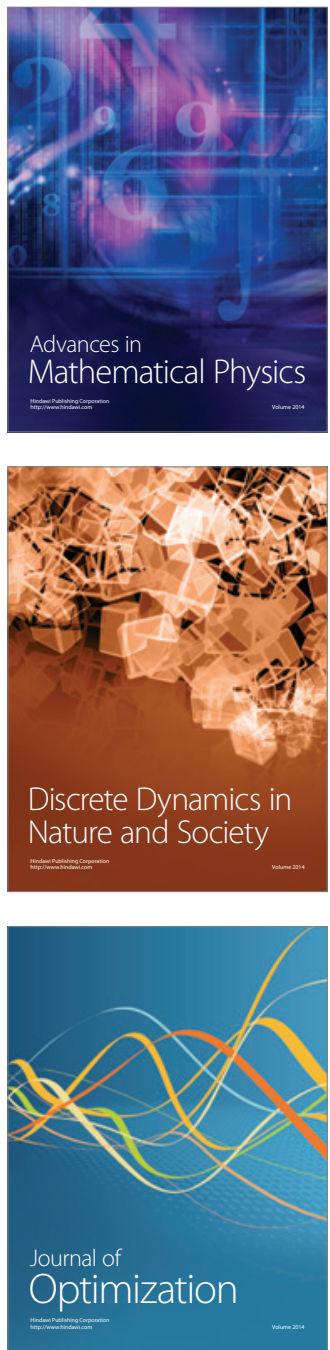